\title{
Adatom Fe(III) on the hematite surface: Observation of a key reactive surface species
}

\author{
Carrick M. Eggleston ${ }^{\text {a) }}$ \\ Department of Geology and Geophysics, University of Wyoming, Laramie, Wyoming 82071-3006 \\ Andrew G. Stack ${ }^{\text {b) }}$ \\ Department of Land, Air and Water Resources, University of California, Davis, California 95616 \\ Kevin M. Rosso ${ }^{\text {c) }}$ \\ William R. Wiley Environmental Molecular Sciences Laboratory, Pacific Northwest National Laboratory, \\ P.O. Box 999, MSIN K8-96, Richland, Washington 99352
}

Angela M. Bice

Department of Geology and Geophysics, University of Wyoming, Laramie, Wyoming 82071-3006

(Received 2 February 2004; accepted 26 May 2004; published online 30 June 2004)

\begin{abstract}
The reactivity of a mineral surface is determined by the variety and population of different types of surface sites (e.g., step, kink, adatom, and defect sites). The concept of "adsorbed nutrient" has been built into crystal growth theories, and many other studies of mineral surface reactivity appeal to ill-defined "active sites." Despite their theoretical importance, there has been little direct experimental or analytical investigation of the structure and properties of such species. Here, we use ex-situ and in-situ scanning tunneling microcopy (STM) combined with calculated images based on a resonant tunneling model to show that observed nonperiodic protrusions and depressions on the hematite (001) surface can be explained as $\mathrm{Fe}$ in an adsorbed or adatom state occupying sites different from those that result from simple termination of the bulk mineral. The number of such sites varies with sample preparation history, consistent with their removal from the surface in low $p \mathrm{H}$ solutions. (C) 2004 American Institute of Physics. [DOI: 10.1063/1.1772991]
\end{abstract}

\section{INTRODUCTION}

Mineral surfaces are the medium upon which the Earth's solids and fluids interact. Their reactivity in the fundamental processes of adsorption, dissolution/growth, and electron transfer is directly tied to their atomic structure. ${ }^{1}$ In addition to two-dimensionally periodic surface structures, there are one-dimensional periodic and zero-dimensional structures such as steps and kink sites that play important roles in mineral reactivity. These include adsorbed or adatom sites. There are long-standing models of surface chemistry that appeal to the existence of populations of adatom species having properties distinct from both the solid and aqueous solutes. In crystal growth theories, ${ }^{2}$ for example, dissolved nutrient is postulated to pass through the "adsorbed nutrient" state before incorporation into the crystal structure. Despite the theoretical importance of a pool of adsorbed nutrient in mineral dissolution and growth, there is little work confirming its existence or properties on mineral oxides. Surface adatom species (or sites) are difficult to study because of their small size, restriction to surfaces and interfaces, and nonperiodic nature. Indirectly, it has been shown that transient spikes in dissolution rate occur in response to $p \mathrm{H}$ changes in a way consistent with the formation and dissolution of adsorbed $\mathrm{Fe}$ at the hematite surface, ${ }^{3-6}$ and an isotopic exchange and

\footnotetext{
${ }^{a)}$ Electronic mail: carrick@uwyo.edu

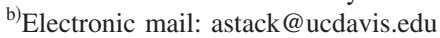

${ }^{c}$ Electronic mail: Kevin.Rosso@pnl.gov
}

Mössbauer study by Rea $e t$ al. ${ }^{7}$ concluded that a population of kinetically labile sites characterizes the ferrihydrite surface.

Nonperiodic adatom sites at mineral surfaces can behave quite differently from other surface sites, and thus are crucial to understanding the overall chemical reactivity of mineral surfaces. Adatom sites are more sterically accessible than other sites and thus may be more easily complexed by organic molecules that are prone to formation of multidentate surface complexes. There is evidence that organic molecule adsorption can be enhanced by formation of organic-Fe ternary surface complexes, which suggests that adsorbed or adatom iron creates a surface more prone to organic molecule adsorption. ${ }^{8,9}$ Here, we make a first step toward direct study of such adsorbed, adatom, or nonperiodic surface material for the case of hematite $\left(\alpha-\mathrm{Fe}_{2} \mathrm{O}_{3}\right)$. We present atomically resolved scanning tunneling microscopy (STM) images of hematite (001) surfaces, supported by a resonant tunneling model (RTM) parametrized with ab initio calculations, that are consistent with the existence of nonperiodic adsorbed $\mathrm{Fe}$ on the periodic hematite (001) surface structure. We also present initial evidence that exposure of the surface to acidic conditions removes much of the nonperiodic material from the surface. This suggests a direct correspondence between the observed nonperiodic $\mathrm{Fe}$ and the dissolution-labile $\mathrm{Fe}$ observed in other studies of hematite. ${ }^{3-6}$

\section{HEMATITE (001) SURFACE STRUCTURE}

Hematite surfaces have been a frequent subject of STM study because hematite is one of the very few common ox- 


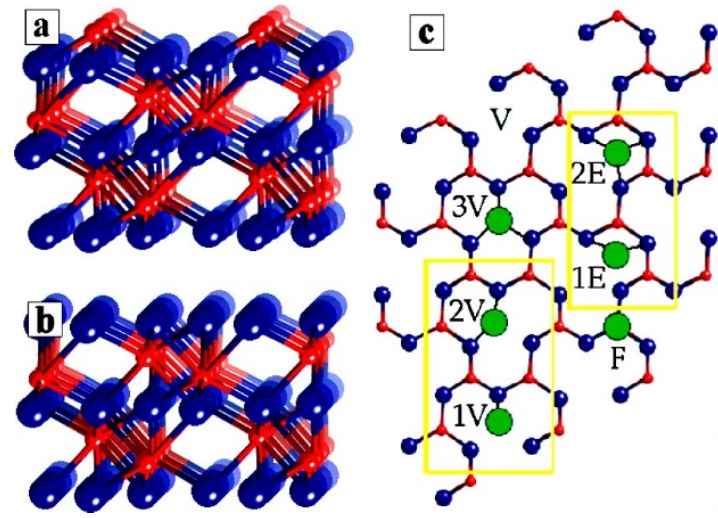

FIG. 1. (Color) Illustrations of hematite (001) surface structures. Blue spheres represent oxygen; in (c), they represent the uppermost oxygen layer. Red spheres represent $\mathrm{Fe}$; in (c) they represent $\mathrm{Fe}$ in "B" and "C" octahedral sites below the uppermost oxygen layer. Every third octahedral site is vacant. Green circles represent $\mathrm{Fe}$ in surface sites above the uppermost oxygen layer. (a) Illustration of the Fe termination; Fe resides in trigonal surface sites over vacancies in the underlying octahedral layer [see the $3 \mathrm{~V}$ sites in panel (c)]. (b) The $\mathrm{O}$ termination; the uppermost $\mathrm{Fe}$ ions in (a) have simply been removed (relaxations, as well as adsorbed $\mathrm{H}_{2} \mathrm{O}$ and its dissociation products are not shown). (c) Illustration of some possible "adatom" or "adsorbed" $\mathrm{Fe}$ sites. The $3 \mathrm{~V}$ site is an Fe over a vacancy (V) in the underlying octahedral layer; this is also called the " $A$ " site and can be part of a bulk termination [identical to the uppermost Fe in panel (a)]. The F site shares an octahedral face with an underlying octahedral Fe, and although it could be part of a bulk termination, such sites have not been observed. The $2 \mathrm{~V}$ and $1 \mathrm{~V}$ sites represent Fe in or near a $3 \mathrm{~V}$ site but with one and two bonds to the surface severed. The $2 \mathrm{E}$ and $1 \mathrm{E}$ sites are $\mathrm{Fe}$ in sites sharing 2 and 1 edge with underlying octahedra. The $2 \mathrm{~V}, 1 \mathrm{~V}, 2 \mathrm{E}$, and $1 \mathrm{E}$ sites (yellow boxes) do not occur as part of a termination of the hematite bulk structure.

ides that is sufficiently conductive for STM. ${ }^{10-19}$ STM results are generally consistent with the existence of two different surface structural domains $(\mathrm{Fe}-$ and $\mathrm{O}$ terminations ${ }^{15-17,19}$ Fig. 1). Hematite has also been used in wet-chemical studies of adsorption and dissolution (including reductive dissolution), allowing for the possibility of relating observed surface structures to reactivity effects or consequences. ${ }^{20-25}$

A resonant tunneling model (RTM) parametrized using $a b$ initio calculations has been used as a way to take solvent reorganization (Marcus theory ${ }^{26-29}$ ) into account in the calculation of STM images for different two-dimensionally periodic surface structures. ${ }^{19}$ Molecular modeling of water adsorption on $\mathrm{Fe}$ - and O-terminated hematite (001) surfaces has also been carried out. ${ }^{30}$ Experimental STM images from previous studies have often revealed a range of nonperiodic surface features (e.g., see Fig. 2). As suggested in Ref. 19, the widespread nature of these nonperiodic features suggests a parallel between them and adsorbed or adatom $\mathrm{Fe}$, but it is necessary to rule out other possibilities and to probe the properties of these features both experimentally and theoretically in order to support such an interpretation.

\section{METHODS}

\section{A. Hematite}

We used natural hematite from Tarascon sur Ariège, France. Sample preparation and characterization are described in more detail in Ref. 19. We used samples that had been exposed to distilled and doubly deionized (DDI) water for $96 \mathrm{~h}$ and either imaged subsequently or exposed to concentrated nitric acid for $1 \mathrm{~h}$ before imaging.

Natural hematite is typically an $n$-type semiconductor at $25^{\circ} \mathrm{C}$ due to donor impurities. Based on resistivity, our hematite had an estimated impurity concentration (donors minus acceptors) of between $1 \times 10^{-3}$ and $4 \times 10^{-2}$ at. \%. A laser-ablation ICP-MS analysis gave a total donor minus acceptor concentration of $2.0 \times 10^{-3}$ at. \%, within the range of values from resistivity. These semiquantitative measurements confirm that our hematite is $n$-type, and show that the main donor impurities are $\mathrm{Sn}$ and Ti. Adding up donor, acceptor, and impurities that do not affect conduction, we have about $6 \times 10^{-3}$ at. \% impurities on the basis of ICP-MS. Oxygen vacancies, which act as electron donors, are included in the resistivity measurement but not in the ICP-MS measurement.

\section{B. Scanning tunneling microscopy (STM)}

A Digital instruments Nanoscope IIIa controller was used with a Molecular Imaging electrochemical STM with a 4- $\mu \mathrm{m}$ scanner to image hematite both in- and ex-situ (in $p \mathrm{H}$ $1 \mathrm{HNO}_{3}$ or DDI $\mathrm{H}_{2} \mathrm{O}$ when in-situ). Imaging conditions (bias voltage, setpoint current) are given in the figure captions. For ex-situ imaging we used electrochemically etched tungsten tips, and for in-situ imaging we used commercial Pt/Ir tips insulated with Apiezon wax and tested for $<10 \mathrm{pA}$ faradaic current (Molecular Imaging).

\section{Resonant tunneling model (RTM)}

The concept of resonant tunneling is well known in solid-state physics, and is the basis of devices known as resonant tunneling diodes. ${ }^{31,32}$ The models have been extended to include thin-film semiconductor devices that are physically akin to the situation described here (metal tip, resonator in the tip-sample gap, semiconductor). As applied to hematite, the RTM is a relatively quick way to evaluate both the distance dependence of current from an adatom to the substrate as well as changes in the degree of adatom solvation associated with changes in bonding to the surface. For example, an $\mathrm{Fe}$ atom with one bond to the surface (e.g., a 1V site in Fig. 1) will likely have a different distance to nearest-neighbor $\mathrm{Fe}$ atoms than a $3 \mathrm{~V}$ (or $\mathrm{A}$ ) site and be coordinated by more inner-sphere water molecules than would an Fe atom in A, B, or $\mathrm{C}$ sites (Fig. 1). These factors, in addition to changes in electronic structure, will affect the kinetics of electron transfer from the surface atom to or from an STM tip.

The RTM, and the $a b$ initio calculations used to support and parametrize it, are described in Ref. 19. Here, we present only the main concepts. The solvent structure around an aqueous $\mathrm{Fe}^{3+}$ ion changes when the $\mathrm{Fe}^{3+}$ is reduced to $\mathrm{Fe}^{2+}$. $\mathrm{Fe}-\mathrm{O}$ bond lengths increase, and the hydrogenbonded structure of outer-sphere water of solvation changes. In this reduction (a process of zero net $\Delta G$ known as selfexchange), the solvent reorganizations required to bring the donor and acceptors states to the same energy is the reorganization energy, $\lambda$. For electron transfer between aqueous $\mathrm{Fe}^{3+}$ and $\mathrm{Fe}^{2+} \lambda$ is relatively large (about $100 \mathrm{kT}$ or 2.6 $\mathrm{eV}^{33}$ at room temperature), making it an important consider- 

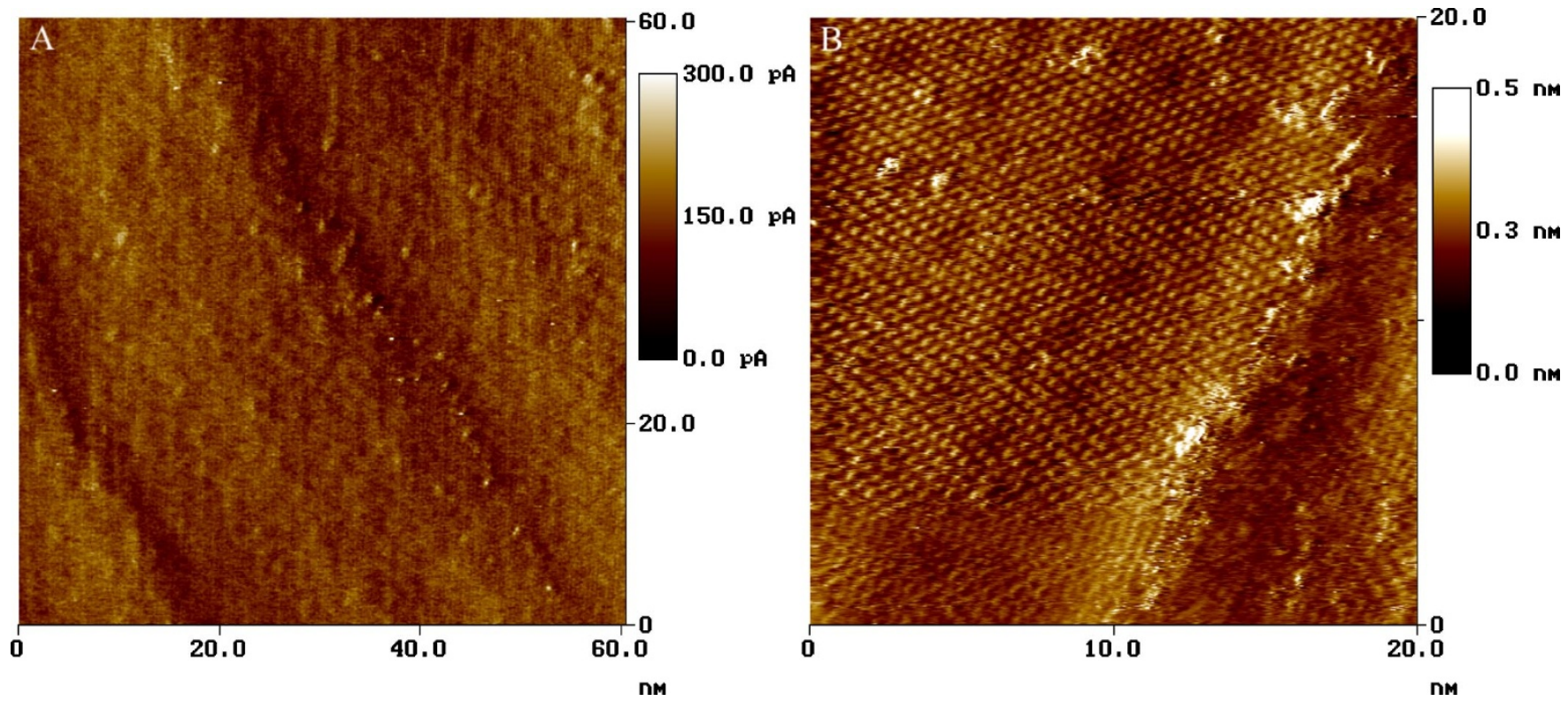

FIG. 2. (Color) Examples of nonperiodic features in STM images of hematite (001) surfaces. (A) A $60 \times 60 \mathrm{~nm}$ image (in air), $-500 \mathrm{mV}, 726 \mathrm{pA}, 300 \mathrm{pA}$ $\mathrm{z}$ range (current contrast). This image shows a semiperiodic modulation of the terrace structure with a $\sim 2 \mathrm{~nm}$ periodicity, as observed in Ref. 19 , and which typically involves height differences between high and low points of about $0.1 \mathrm{~nm}$. In Ref. 19 it was concluded that the higher parts of the modulation are domains of Fe termination. Atomic-scale structure can be discerned in some parts of the image. There are a few nonperiodic bumps on the terraces, but they are most common along the steps. (B) A $20 \times 20 \mathrm{~nm}$ image (in air), $-800 \mathrm{mV}, 500 \mathrm{pA}, \mathrm{z}$ range $0.5 \mathrm{~nm}$. In this image there is a highly periodic surface structure punctuated both by a few protruding "bumps" as well as slight depressions. Again, there are more of these features along the step.

ation for electron transfer to and from hematite in air or water. Methods for calculating current from an electrode (such as an STM tip) through a redox-active monolayer to a metal substrate in which the redox-active layer is subject to solvent reorganization have been presented and tested. ${ }^{34-36}$ We have used this idea because the $\lambda$ for different surface sites (A, B, and $\mathrm{C}$ sites as well as "adsorbed" $\mathrm{Fe}$ in sites other than A, B, and C; Fig. 1) can be substantially different from one another because of differing degrees of solvation, leading to different electron-transfer characteristics for different surface sites.

We only consider tunneling to and from iron atoms, in agreement with previous work. ${ }^{16,19,37}$ The $\mathrm{A}, \mathrm{B}$, and $\mathrm{C}$ sites, as well as nonperiodic or adatom sites, are the resonators mediating electron transfer between the tip and the hematite substrate. Electron transfer (ET) occurs in two steps; for current from substrate to tip, ET occurs first from the substrate to the redox center $(s r)$, and then from the redox center to the tip $(r t)$. We assume that the density of states of substrate and tip are independent of bias voltage. The tunneling current, $j$, is then

$$
j \propto-e_{0} \frac{\pi}{\hbar} \exp \left(-\beta_{r t} d_{r t}\right) \exp \left(-\beta_{s r} d_{s r}\right) \int_{0}^{e V_{b}} D_{o x}(\varepsilon) d \varepsilon,
$$

where $\beta$ is the tunneling decay constant for the couple indicated by the subscript, $d$ is the distance between the designated couple (the nearest-neighbor $\mathrm{Fe}$ atoms are considered the substrate, which determines the $d_{s r}$ distances), $\varepsilon$ is the electron energy, and $V_{b}$ is the STM sample bias voltage relative to the tip. $e_{0}$ and $\hbar$ are the electron charge and Planck's constant, respectively. For given distances and electronic structures, the current is thus proportional to the density of unoccupied states on the resonator, also called the density of oxidized states $D_{o x}(\varepsilon)$, which can be approximated by a Gaussian

$$
D_{o x}(\varepsilon)=\sqrt{\frac{\pi}{k T \lambda}} \exp \left[\frac{-\left(\varepsilon-\varepsilon_{r}\right)^{2}}{4 \lambda k T}\right],
$$

where $\lambda$ is the Marcus reorganization energy, ${ }^{26}$ and $\varepsilon_{r}$ is the reduction potential of the redox center. The $\lambda$ term allows us to incorporate the effect of a solvent on current through surface sites. $\lambda$ can be separated into inner-sphere $\left(\lambda_{I S}\right)$ and outer-sphere $\left(\lambda_{O S}\right)$ components. ${ }^{27}$

Unlike studies in which bias voltage was kept at a constant, small value and only the electrode potential was varied relative to a reference electrode, ${ }^{36,38}$ here the bias voltage is varied. The resulting image characteristics are therefore

TABLE I. Parameters used in the RTM. Other symbols are defined in the text, except "NN" which indicates the number of equivalent "nearest neigbors."

\begin{tabular}{cccccccc}
\hline \hline & $\begin{array}{c}\beta_{s r} \\
\left(\AA^{-1}\right)\end{array}$ & $\begin{array}{c}\beta_{r t} \\
\left(\AA^{-1}\right)\end{array}$ & $\begin{array}{c}d_{s r} \\
(\AA)\end{array}$ & $\begin{array}{c}\lambda_{i s} \\
(\mathrm{eV})\end{array}$ & $\begin{array}{c}\lambda_{o s} \\
(\mathrm{eV})\end{array}$ & $\begin{array}{c}\varepsilon_{r} \\
(\mathrm{eV})\end{array}$ & $\mathrm{NN}$ \\
\hline $\mathrm{A}$ & 1.2 & 1.2 & 3.550 & 0.474 & 1.686 & 0.40 & 1 \\
$\mathrm{~A}$ & 1.2 & 1.2 & 3.290 & 0.474 & 1.686 & 0.40 & 3 \\
$\mathrm{~B}, \mathrm{C}$ & $1.0 / 1.2$ & $1.0 / 1.2$ & 2.959 & 0.274 & 0.126 & 0.00 & 3 \\
\hline \hline
\end{tabular}




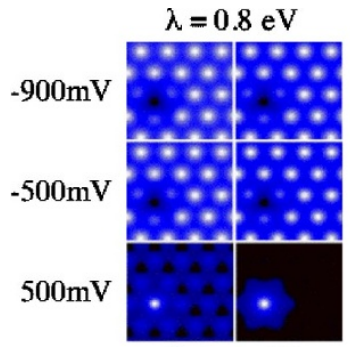

0

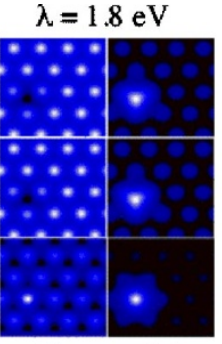

0.1

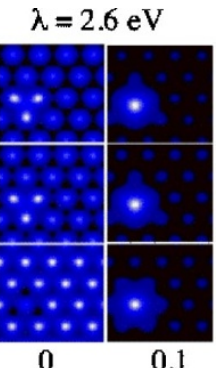

0.1
FIG. 3. (Color) Images calculated using the RTM for different bias voltages (listed at left), reorganization energies ( $\lambda$; list across the top for each block of six images), and $\mathrm{Fe}$ atom heights (listed across the bottom, in $\AA$ ). These images show local current with the tip at constant height.

somewhat more complex than the simple increases and decreases in current when a particular site comes into and then out of resonance. However, our approach is in keeping with the simple STM imaging we performed, rather than electrochemical resonant tunneling microscopy. We discuss some of these complexities as the calculated images are presented and compared to STM images.

To calculate images, we need values for the parameters in Eqs. (1) and (2) for each type of site we wish to model. Plane-wave pseudopotential calculations and density functional theory calculations on clusters were used to predict bond lengths and thus $d_{s r}, \lambda\left(=\lambda_{I S}+\lambda_{O S}\right), \varepsilon_{r}$, and contributed to the determination of $\beta$. Details on the calculations and the methods used can be found in Ref. 19. For the case of "adsorbed" Fe, we have not attempted to model each of the many different structures that could occur. Instead, we simply recognize that the various possible sites will have a range of $\lambda, d_{r t}, d_{s r}$, and $\varepsilon_{r}$ values. We initially use $\varepsilon_{r}$ from the A sites modeled in Ref. 19 (see Fig. 1) and vary the height of the Fe site above the surface (which varies $d_{s r}$ and $d_{r t}$ ) as well as the $\lambda$ attributed to the site. We also vary $\varepsilon_{r}$ at fixed $\lambda$. This approach allows us to test a range of conditions in a simple way, but cannot be expected to model all of the possible structural variations for such adatoms. In effect, the RTM as applied here is a qualitative guide to trends, but cannot be expected to quantitatively reproduce the experimental results.

The parameters used in the RTM are give in Table I. We used a model in which spin states determine whether neighboring Fe sites can contribute electrons to the surface sites in question (see Ref. 19). We adjusted the height of the tip in the RTM for each set of conditions until the average tunneling current was the same for each calculated image; this most closely approximates conditions of constant-height STM imaging at a setpoint current.

\section{RESULTS AND DISCUSSION}

\section{A. RTM calculations}

Figure 3 summarizes the results of a series of RTM calculations for surface $\mathrm{Fe}$ "A" sites (Fig. 1) with different reorganization energies $(\lambda)$ and heights above the surface plane relative to those in the rest of the surface structure, as described in Sec. III. The location of this site is clear in Fig. 3 , and is the same for other calculated images presented be- low. Compared to A-type surface sites with $\lambda \approx 2.2 \mathrm{eV}$, adatoms with smaller $\lambda$ appear as bumps at positive bias and depressions at negative bias. However, for surface sites with larger $\lambda$ than other surface sites the results are reversed, with a depression at positive bias and slight protrusions at negative bias (the triplet is an additive effect, discussed later). Calculated images are also presented in Fig. 3 for an adatom that is $0.1 \AA$ higher above the surface than an A site. This calculation is an attempt to mimic the possible effect of $2 \mathrm{~V}$, $1 \mathrm{~V}$, and $2 \mathrm{E}$ sites (Fig. 1) in which Fe atoms may sit slightly higher above the surface than in a $3 \mathrm{~V}$ site. In this case, protrusions are observed under all conditions except negative bias and low $\lambda$. A general conclusion is that both protrusions and depressions can be observed at a given bias voltage, depending on the $\lambda$, height, and $\varepsilon_{r}$ that characterize each site. Other RTM results are presented in comparison with specific images below.

\section{B. Bias voltage and preparation dependence of STM images}

Through acquisition of hundreds of images of hematite (001) surfaces prepared in various ways, we noted a tendency for nonperiodic features to be observed mostly at higher negative bias voltages for which electrons tunnel from the sample surface to the STM tip. Figure 4 gives one observation of this general result. Although there are undulations on the surface for all images, the bright nonperiodic features that extend roughly a monolayer above the surface in these height images only occur at $-800,-700$, and $-600 \mathrm{mV}$. In other imaging sessions, we sometimes observed nonperiodic features at other bias voltages, but the general observation is that more nonperiodic features are observed at higher negative bias.

Figure 5 demonstrates this point and also shows the importance of sample preparation history. Two data sets are presented, one for a surface that had been soaked in DDI water for about $96 \mathrm{~h}$ before imaging in air (blue squares) and one for a surface that had been boiled in concentrated nitric acid for $1 \mathrm{~h}$ before imaging in air. A relatively large number of nonperiodic features are observed at -400 to $-800 \mathrm{mV}$ on the water-soaked sample, but almost none was observed on the acid-treated sample. For Fig. 5, we used a height threshold of $0.23 \mathrm{~nm}$ (the height of a monolayer step; nonperiodic features smaller than this were omitted from the count along with current "spikes"), which omits depressions [e.g., see Fig. 2(b)] from the overall count. Note, however, that the water-soaked sample had been boiled in nitric acid prior to the water treatment as part of cleaning procedures, indicating that the nonperiodic surface features can be regenerated by aging in water. This behavior is similar to the observed regeneration of "active" of kinetically labile dissolution sites by near-neutral $p \mathrm{H}$ solutions observed in Ref. 5 . Because water adsorbs to the hematite surface from air, it is likely that an acid-treated surface gradually reverts to a state with more nonperiodic material during storage in air. This in turn suggests that $\mathrm{Fe}(\mathrm{III})$ is labilized on the hematite surface over a period of several days, although a more precise kinetic study is needed. 

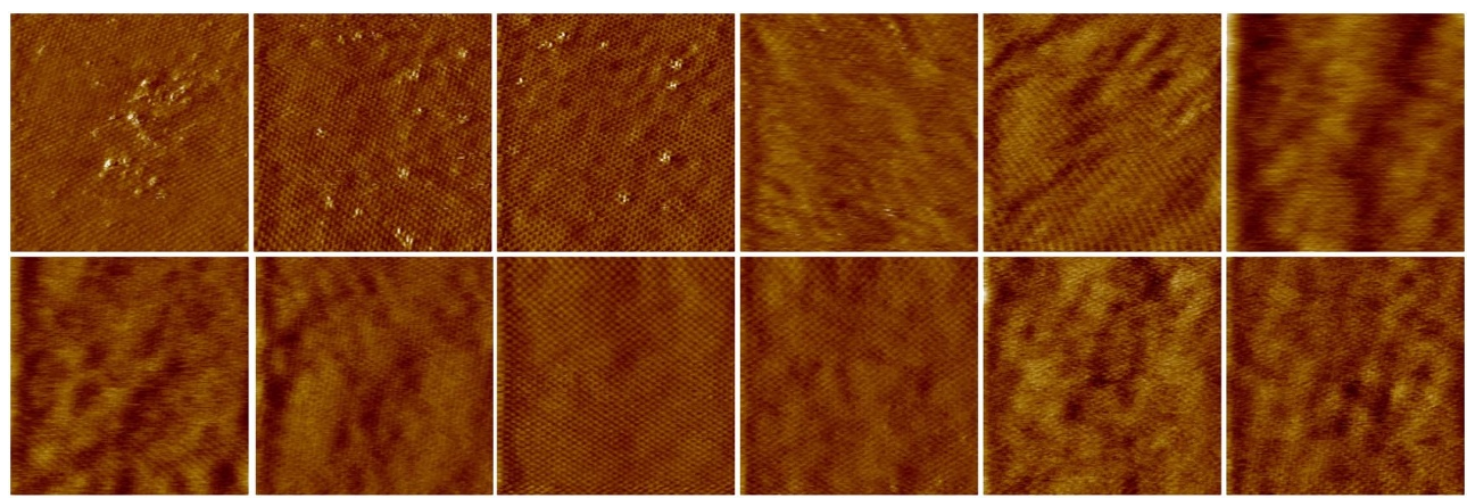

FIG. 4. (Color) STM images of the hematite (001) surface, all taken during one imaging session within a 3-h period using one tip. All images were taken at a setpoint current of $500 \mathrm{pA}$, and all are displayed with a color scale of $0.3 \mathrm{~nm}$. Top row $(\mathrm{mV})$ : $-800,-700,-600,-500,-300,-100$. Bottom row (mV): 100, 200, 300, 400, 600, 800. All images are $20 \times 20 \mathrm{~nm}$.

Figure 6 demonstrates observation of nonperiodic sites both as a function of time and of bias voltage during a single imaging session in $p \mathrm{H} 1 \mathrm{HNO}_{3}$. We present a series of images in Fig. 6 in which, initially, we alternate between low and high negative bias voltage. At $-300 \mathrm{mV}$ [Fig. 6(a)], undulations but no atomic-scale nonperiodic sites are evident; increasing the bias voltage to $-778 \mathrm{mV}$ [Fig. 6(b)] brings out nonperiodic sites, and a return to $-300 \mathrm{mV}$ [Fig. 6(c)] makes them less (or not) evident. Nonperiodic sites are then again observed at $-800 \mathrm{mV}$ [Fig. 6(d)], but not at $-400 \mathrm{mV}$ [Fig. 6(e)]. At $-600 \mathrm{mV}$, nonperiodic sites are again evident after about $1.5 \mathrm{~h}$ of imaging. After $2.5 \mathrm{~h}$ of imaging at $p \mathrm{H} \mathrm{1,} \mathrm{however,} \mathrm{nonperiodic} \mathrm{sites} \mathrm{are} \mathrm{not} \mathrm{evident,}$ even at high negative bias voltages of up to $-1.0 \mathrm{~V}$ [Figs. $6(\mathrm{~g})-(\mathrm{i})]$. This suggests that the surface species that are imaged as the nonperiodic sites may have dissolved in the $p \mathrm{H} 1$ solution during imaging. This interpretation is consistent

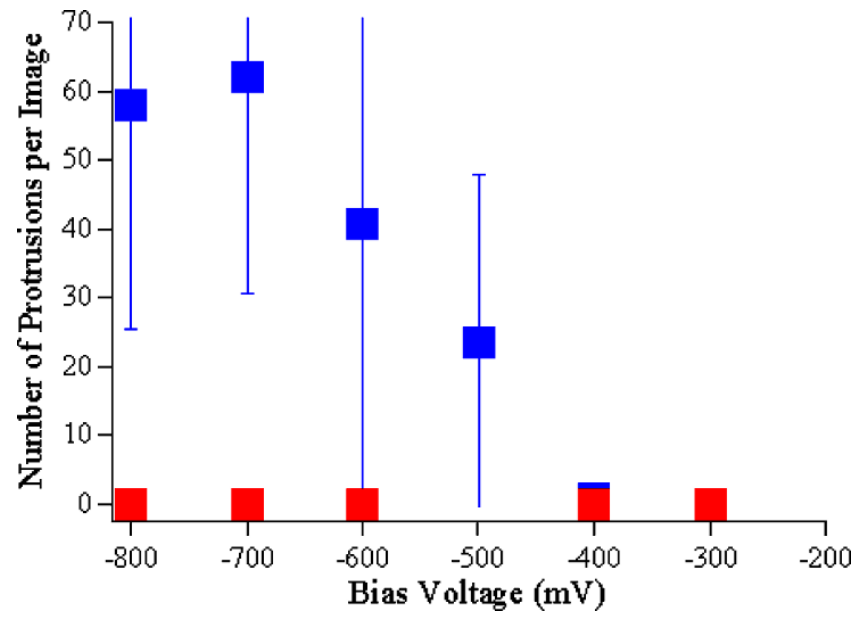

FIG. 5. (Color) Number of nonperiodic sites (protrusions of height greater than $0.23 \mathrm{~nm}$ ) per image, from a series of 142 images, all of the same size $(20 \times 20 \mathrm{~nm})$. "Spikes" in current that only affect one or two pixels of a single scan line were also omitted. For a surface prepared by aging for $96 \mathrm{~h}$ in DDI $\mathrm{H}_{2} \mathrm{O}$ (but imaged in air), relatively large numbers of nonperiodic sites were observed in the -800 to $-500 \mathrm{mV}$ range but not at other bias voltages (blue squares). For a surface prepared by boiling in nitric acid for 1 $\mathrm{h}$, nonperiodic sites were not observed in any significant numbers (red squares). The data points represent averages for different images; error bars are $2 \sigma$ values from the variance in numbers of sites for the different images. with the macroscopically observed accelerated dissolution of a kinetically labile form of $\mathrm{Fe}$ at the hematite surface over a period of a few hours in response to $p \mathrm{H}$ jumps. ${ }^{3,4} \mathrm{We}$ emphasize that we do not think that the nonperiodic sites are created by high negative bias; our interpretation is that high negative bias is a necessary condition for significant tunneling current to flow through these sites. This point is illustrated in the next section. Also, we point out that steady-state hematite dissolution rates at $p \mathrm{H} 1$ (see Ref. 5) correspond to only one Fe atom removed from a $20 \times 20 \mathrm{~nm}$ area every 12 $\mathrm{h}$ on average. This strongly suggests that the apparent dissolution of the nonperiodic sites indicates the dissolution of a kinetically labile form of surface Fe akin to that observed by wet-chemical means in other studies. ${ }^{5-7}$

\section{Dual-bias imaging}

Images taken in sequence are open to the criticism that either (a) different places with different surface features are imaged as time passes (because of drift and tip variability, it is virtually impossible to image in one location at the atomic scale for more than a few scans); or (b) the STM tip causes changes in the surface, possibly by sweeping protrusions aside as successive scans are made, eventually making an area appear "clean" (although we point out that the images in Fig. 4 were not taken in sequence from $-800 \mathrm{mV}$ on up to $+800 \mathrm{mV}$, but in a more random order). Dual-bias imaging allows us to obtain two images, at different bias voltages, of the same place simultaneously (every other scan line is taken at a different bias voltage). Figure 7 gives three examples of such imaging in which the negative bias image shows nonperiodic surface features that are not evident in the positive bias scan of the same area. This observation is in agreement with Figs. 4-6, and with them indicates that nonperiodic surface features are evident at -500 to $-800 \mathrm{mV}$ bias that are not evident at positive bias.

This situation is most closely matched in the RTM calculations (Fig. 3) by conditions of high reorganization energy $(\lambda)$. However, Fig. 7 does not show that the protrusions in the negative bias image always correspond to depressions in the positive bias image. Figure 8 shows RTM calculations for different resonance energies $\left(\varepsilon_{r}\right)$ for $\lambda$ fixed at $2.3 \mathrm{eV}$ in 

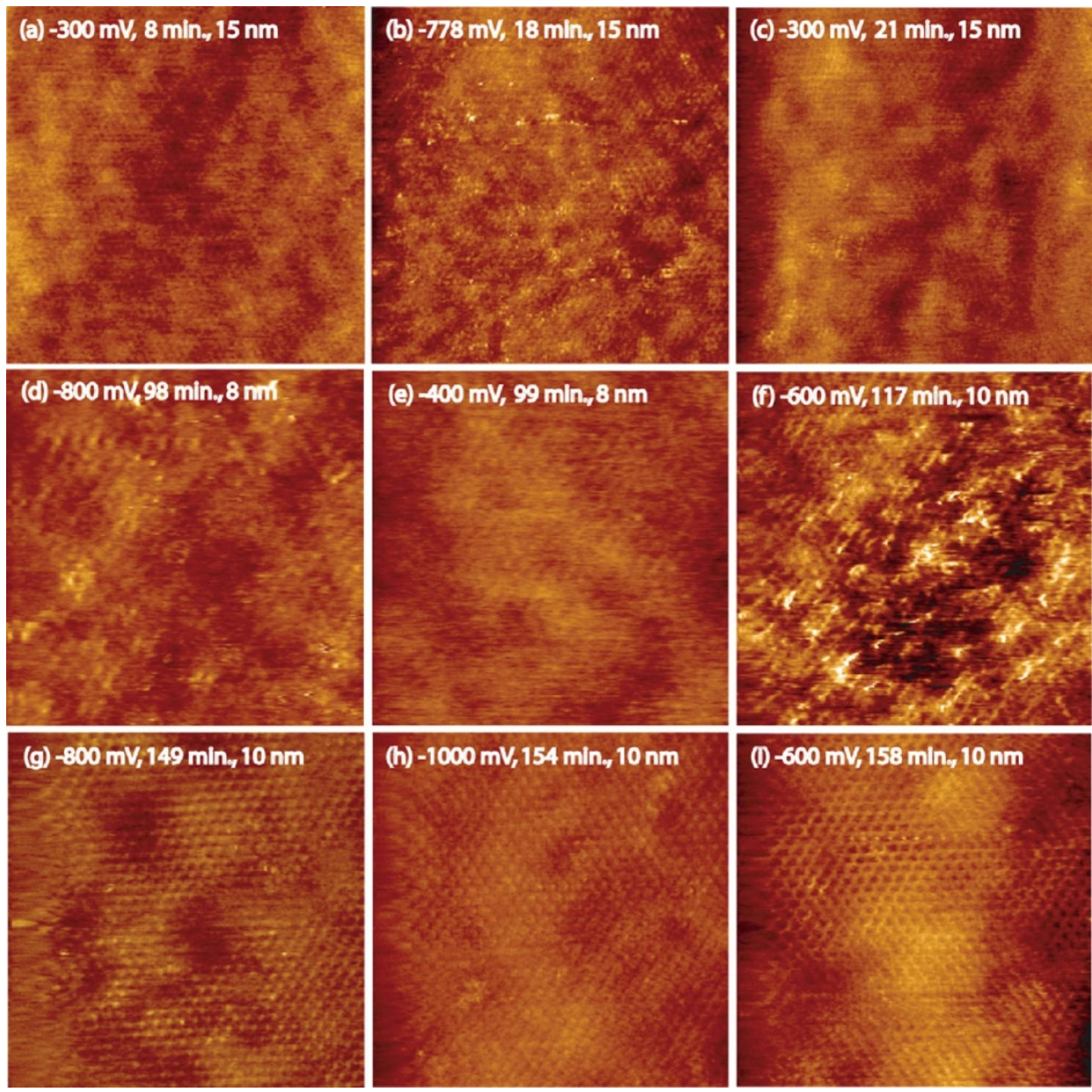

FIG. 6. (Color) A sequence of STM images of hematite (001) taken in-situ in $p \mathrm{H} 1 \mathrm{HNO}_{3}$ at room temperature using insulated tips. Bias voltages, image size (e.g., $15 \mathrm{~nm}=15 \times 15 \mathrm{~nm}$ image) and acquisition time are given in the figure. All images are height images with a vertical color scale of 0.3 $\mathrm{nm}$. Nonperiodic sites are observed mostly at higher negative bias and earlier in the experiment. After over $2 \mathrm{~h}$ from the start of the experiment, nonperiodic surface sites are less common, particularly those that protrude more than $0.23 \mathrm{~nm}$, even at higher negative bias voltage $[(\mathrm{g})-(\mathrm{i})]$. which protrusions in the negative-bias images are accompanied by only a slight depression in the positive bias image. Figure 7 also shows, in addition to protrusions, depressions that are found in both the negative and positive bias images. Figure 9 shows that this can be produced in the calculated images for conditions of low $\lambda$ but high resonance energy.

Although the RTM cannot be regarded as quantitatively accurate, the RTM results presented in Figs. 3, 8, and 9 suggest that the STM observations in Fig. 7 might be explained if the local $\lambda$ and resonance energies of the sites involved differ slightly from those of the periodic surface structure. It should also be kept in mind that the RTM calculations only consider an A-type site with altered RTM parameters on an iron-terminated surface; the behavior of Fe adatoms on an oxygen-terminated surface is likely to be quite different.

\section{Structural hints}

Figure 3 shows triplet sites at higher negative bias for A sites with $\lambda$ greater than that of a normal A site. Triplets are an additive effect in which, when the tip is positioned over the B site, the total current is the normal B site current plus some current added from increased current through the A site, making the B site appear larger. Although such triplets are peculiar to an A site with an altered $\lambda$ parameter, it is possible that an $\mathrm{Fe}$ atom near an $\mathrm{A}$ site would produce a triplet site in STM images, perhaps not with all members of the triplet of equal height if the $\mathrm{Fe}$ is not directly over the A site. Such an observation could also be explained as a cluster of three iron ions in A sites. Triplets are indeed observed in some STM images, along with double and single sites. Figure 10 is a particularly striking example. Usually, in STM images, the occurrence of multiple examples of the same pattern is evidence for a multiple-tip artifact rather than a real surface structure, but in Fig. 10 not all of the triplets "point" in the same direction as they must for a multiple-tip artifact. While most triplets "point" to the left (blue arrows mark many but not all of these), some "point" to the right (red arrows). This demonstrates that the triplets are not a tip artifact.

\section{E. Impurities and site densities}

The measured impurity concentration allows us to ask whether the nonperiodic bumps and depressions in the STM images could be caused by impurity atoms (e.g., through local potential effects on the local electronic structure). For example, in a $20 \times 20 \mathrm{~nm}$ STM image, there are just under $5500 \mathrm{Fe}$ sites (assuming an Fe termination and including A, $\mathrm{B}$, and $\mathrm{C}$ sites). Based on our highest impurity concentration $\left(4 \times 10^{-2}\right.$ at. $\left.\%\right)$, only about 2.2 atoms are impurities. As pointed out in Eggleston et al. (2003), we have observed up to about 60 nonperiodic features in some $20 \times 20 \mathrm{~nm}$ images. 


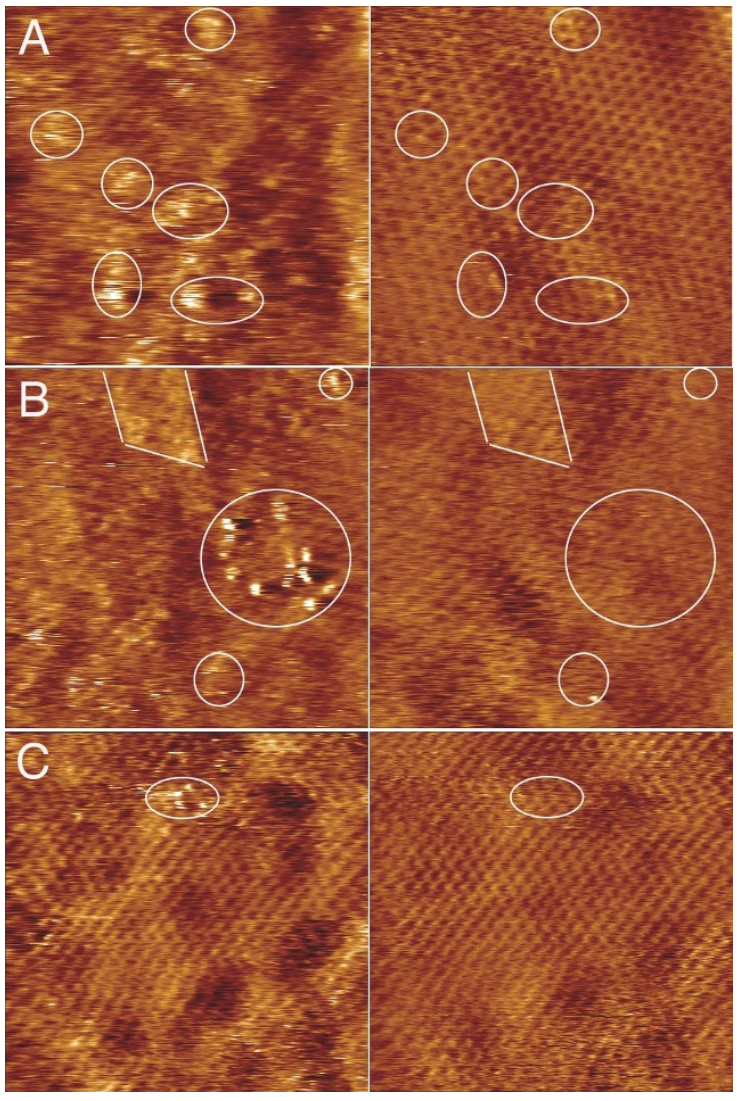

FIG. 7. (Color) Dual-bias STM images of the hematite (001) surface, taken in air. All images have a $\mathrm{z}$ range of $0.30 \mathrm{~nm}$, and all are $14 \times 14 \mathrm{~nm}$. (A) -700 and $+600 \mathrm{mV}, 424 \mathrm{pA}$; (B) -800 and $+500 \mathrm{mV}, 424 \mathrm{pA}$; (C) -600 and $+400 \mathrm{mV}, 700 \mathrm{pA}$. Circles and lines have been overlaid on all images to aid comparison of features in the negative-bias and positive-bias images.

Therefore, only a small minority of the nonperiodic features can be explained by appeal to impurities exclusively, and it is therefore highly unlikely that most of the observed nonperiodic sites are caused by impurities.

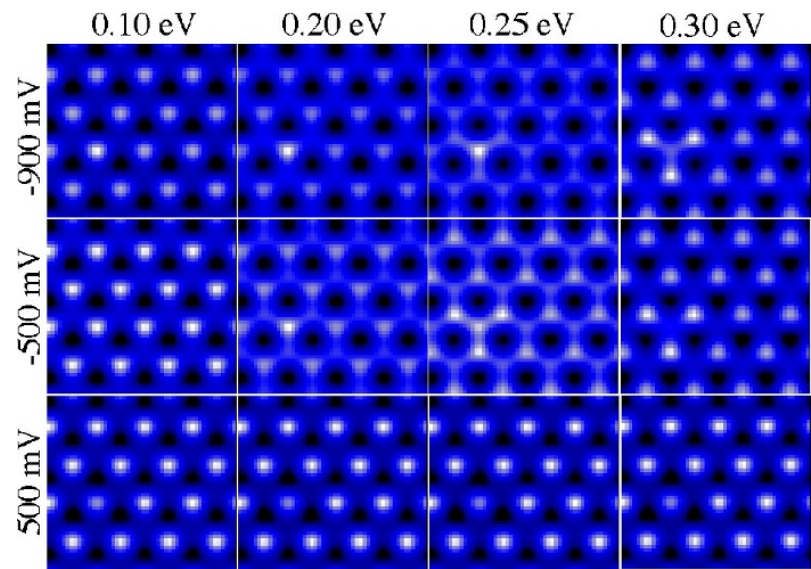

FIG. 8. (Color) Images calculated for bias voltages of $-900,-500$, and $500 \mathrm{mV}$ (on left) at a series of resonance energies $\left(\varepsilon_{\mathrm{r}}\right)$ (heading each column). All calculations were made with $\lambda=2.3 \mathrm{eV}$. The sequence shows a transition from an A site dominated image at low $\varepsilon_{\mathrm{r}}$ to a B site dominated image at higher $\varepsilon_{\mathrm{r}}$. This leads to the triplet sites in Fig. 3 for $\varepsilon_{\mathrm{r}}$ $=0.400 \mathrm{eV}$.

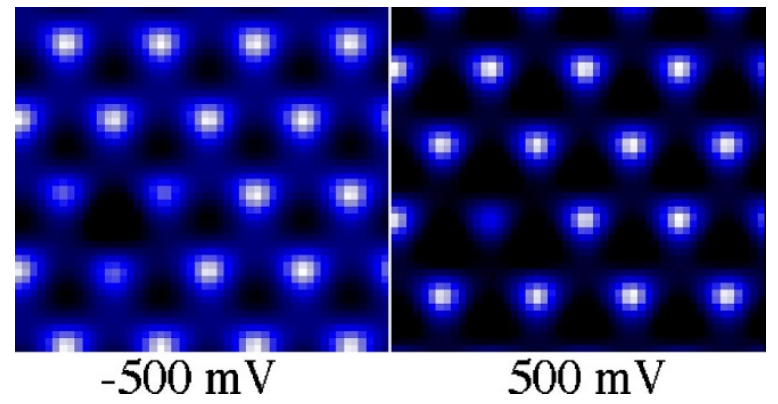

FIG. 9. (Color) RTM calculations made with a $\lambda$ of $0.8 \mathrm{eV}$ and an $\varepsilon_{\mathrm{r}}$ of 0.64 eV.

Another useful comparison between Ref. 4 and the present work is that of site densities. On the basis of dissolution transients in response to $p \mathrm{H}$ jumps, Samson et al. ${ }^{4}$ estimated a site density for kinetically labile surface Fe sites of about $1.0 \mu \mathrm{mol} \mathrm{m}^{-2}$. This translates to about 24 sites in each $20 \times 20 \mathrm{~nm}$ image. Figure 5 shows that our STM observations are up to a factor or 2 to 3 higher than this, but are within the same order of magnitude.

\section{CONCLUSIONS}

We have presented calculated and experimental STM images showing that nonperiodic sites (both protrusions and depressions) observed on hematite (001) surfaces by STM, both ex-situ and in-situ, are consistent with $\mathrm{Fe}$ in an adsorbed or adatom state on the hematite surface. These sites are observed as protrusions at higher negative bias voltages. Acid treatment of the surfaces both prior to imaging and during in-situ imaging removes most of these sites, consistent with their interpretation as adsorbed species. The time scale of

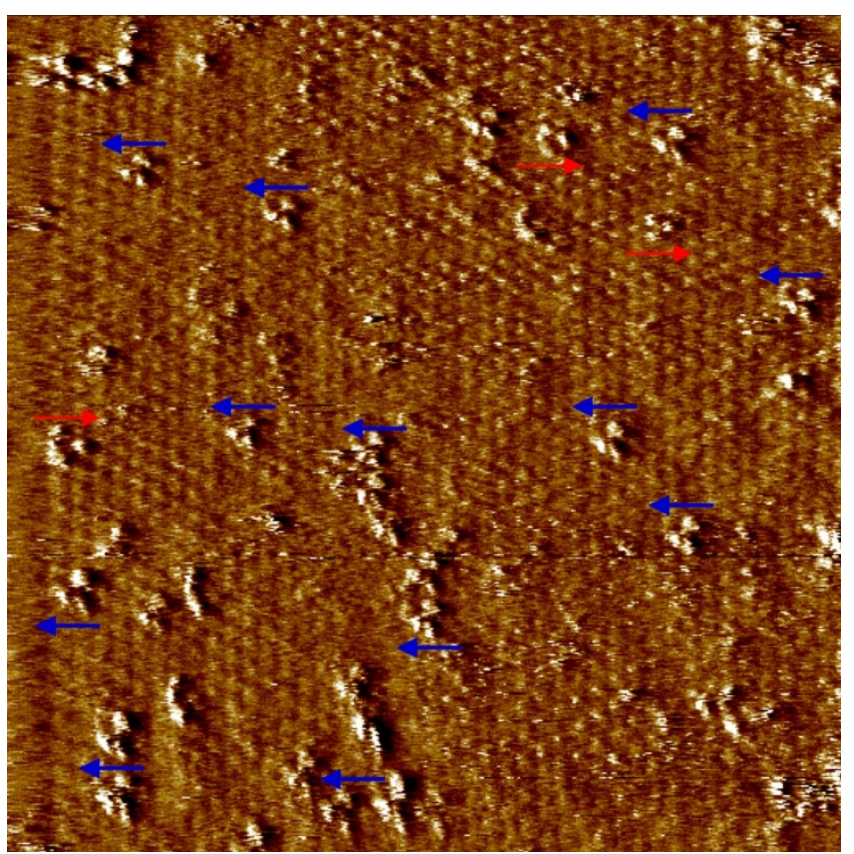

FIG. 10. (Color) STM image, $20 \times 20 \mathrm{~nm},-600 \mathrm{mV}, 500 \mathrm{pA}$. This sample had been soaked in room-temperature DDI water for $96 \mathrm{~h}$ prior to imaging in air. Blue arrows mark many of the nonperiodic triplet sites that "point" to the left; red arrows mark those triplets that "point" to the right. 
removal during in-situ imaging at $p \mathrm{H} 1$ is consistent with the $p \mathrm{H}$-jump experiments of Samson et al. ${ }^{4}$ for dissolution of kinetically labile Fe from the hematite surface during approach to steady-state dissolution, and is roughly consistent with the time scale of isotopic exchange experiments of Rea et $a .^{7}$ for kinetically labile surface Fe on ferrihydrite.

Adatom Fe in nonperiodic and nonbulk sites at the hematite surface represents structures whose chemical behavior differs from, and cannot be directly predicted from, an understanding of the bulk termination structure. The conditions that influence or control the production and consumption of this reservoir of reactive surface sites should thus be a subject of future investigation.

${ }^{1}$ G. E. Brown, Jr. et al., Chem. Rev. (Washington, D.C.) 99, 77 (1998).

${ }^{2}$ J.-W. Zhang and G. H. Nancollas, in Mineral-Water Interface Geochemistry, edited by M. F. Hochella, Jr. and A. F. White, Reviews in Mineralogy Vol. 23 (Mineralogical Society of America, Washington, DC, 1990), pp. 365-396.

${ }^{3}$ S. D. Samson and C. M. Eggleston, Environ. Sci. Technol. 32, 2871 (1998).

${ }^{4}$ S. D. Samson, L. L. Stillings, and C. M. Eggleston, Geochim. Cosmochim. Acta 64, 3471 (2000).

${ }^{5}$ S. D. Samson and C. M. Eggleston, Geochim. Cosmochim. Acta 64, 3675 (2000).

${ }^{6}$ S. D. Samson and C. M. Eggleston, in Water-rock Interaction, Ore Deposits, and Environmental Geochemistry: A Tribute to David A. Crerar, edited by R. Hellmann and S. A. Wood (Geochemical Society, St. Louis, MO, 2001) pp. 61-73.

${ }^{7}$ B. A. Rea, J. A. Davis, and G. A. Waychunas, Clays Clay Miner. 42, 23 (1994).

${ }^{8}$ S. M. Kraemer and J. G. Hering, Geochim. Cosmochim. Acta 61, 2855 (1997).

${ }^{9}$ W. Fish, Portland State University, personal communication, 2004.

${ }^{10}$ M. F. Hochella, Jr., C. M. Eggleston, V. B. Elings, G. A. Parks, G. E. Brown, Jr., C. M. Wu, and K. Kjoller, Am. Mineral. 74, 1233 (1989).

${ }^{11}$ C. M. Eggleston and M. F. Hochella, Jr., Am. Mineral. 77, 911 (1992).

${ }^{12}$ N. G. Condon, P. W. Murray, F. M. Leibsle, G. Thornton, A. R. Lennie, and D. J. Vaughan, Surf. Sci. Lett. 310, L609 (1994).
${ }^{13}$ N. G. Condon, F. M. Leibsle, A. R. Lennie, P. W. Murray, D. J. Vaughan, and G. Thornton, Phys. Rev. Lett. 75, 1961 (1995).

${ }^{14}$ N. G. Condon, F. M. Leibsle, A. R. Lennie, P. W. Murray, T. M. Parker, D. J. Vaughan, and G. Thornton, Surf. Sci. 397, 278 (1998).

${ }^{15}$ X.-G. Wang, W. Weiss, S. K. Shaikhutdinov, M. Ritter, M. Petersen, F. Wagner, R. Schlogl, and M. Scheffler, Phys. Rev. Lett. 81, 1038 (1998).

${ }^{16}$ C. M. Eggleston, Am. Mineral. 84, 1061 (1999).

${ }^{17}$ S. K. Shaikhutdinov and W. Weiss, Surf. Sci. Lett. 432, L627 (1999).

${ }^{18}$ K. Adib, N. Camillone, III, J. P. Fitts, K. T. Rim, G. W. Flynn, S. A. Joyce, and R. M. Osgood, Surf. Sci. 497, 127 (2002).

${ }^{19}$ C. M. Eggleston, A. G. Stack, K. M. Rosso, S. R. Higgins, A. M. Bice, S. W. Boese, R. D. Pribyl, and J. J. Nichols, Geochim. Cosmochim. Acta 67, 985 (2003).

${ }^{20}$ S. Banwart, S. Davies, and W. Stumm, Colloids Surf. 39, 303 (1989).

${ }^{21}$ P. A. Maurice, M. F. Hochella, Jr., G. A. Parks, and G. Sposito, Clays Clay Miner. 43, 29 (1995).

${ }^{22}$ S. J. Hug, J. Colloid Interface Sci. 188, 415 (1997).

${ }^{23}$ J. J. Lenhart and B. D. Honeyman, Geochim. Cosmochim. Acta 63, 2891 (1999).

${ }^{24}$ O. W. Duckworth and S. T. Martin, Geochim. Cosmochim. Acta 65, 4289 (2001).

${ }^{25}$ I. Kristl I. and R. Kretzschmar, Geochim. Cosmochim. Acta 65, 3435 (2001)

${ }^{26}$ R. A. Marcus, J. Chem. Phys. 24, 966 (1956).

${ }^{27}$ R. A. Marcus, J. Chem. Phys. 43, 679 (1965).

${ }^{28}$ R. A. Marcus and N. Sutin, Biochim. Biophys. Acta 811, 265 (1985).

${ }^{29}$ R. A. Marcus, J. Phys. Chem. 94, 1050 (1990).

${ }^{30}$ F. Jones, A. L. Rohl, J. B. Farrow, and W. van Bronswijk, Phys. Chem. Chem. Phys. 2, 3209 (2000).

${ }^{31}$ E. Diez, F. Dominguez-Adame, and A. Sanchez, J. Appl. Phys. 77, 4816 (1995).

${ }^{32}$ C. Presilla and J. Sjostrand, J. Math. Phys. 37, 4816 (1996).

${ }^{33}$ M. Grätzel, Heterogeneous Photochemical Electron Transfer (CRC Press, Boca Raton, FL, 1989).

${ }^{34}$ W. Schmickler and C. Widrig, J. Electroanal. Chem. 336, 213 (1992).

${ }^{35}$ W. Schmickler, Surf. Sci. 295, 43 (1993).

${ }^{36}$ W. Schmickler and N. Tao, Electrochim. Acta 42(18), 2809-2815 (1997).

${ }^{37}$ G. Tarrach, D. Burgler, T. Schaub, R. Wiesendanger, and H. J. Guntherodt, Surf. Sci. 285, 1 (1993).

${ }^{38}$ N. J. Tao, Phys. Rev. Lett. 76, 4066 (1996). 